\title{
ELECCIONES Y PROCESO DE TRANSICIÓN EN CHILE
}

\author{
GUSTAVO PALOMARES LERMA \\ Profesor Titular de Relaciones Internacionales \\ UNED
}




\section{SUMARIO}

Elecciones Y PROCESO de tRANSiCión EN ChILE.-Elecciones 1989: Tabla 1. Computos generales. Tabla 2. Composición de las CAMARAS. 
Revista de Derecho Político, núm. 33, 1991, pp. 477-496

\title{
ELECCIONES Y PROCESO DE TRANSICIÓN EN CHILE
}

\author{
POR \\ GUSTAVO PALOMARES LERMA \\ Profesor Titular de Relaciones Internacionales \\ Departamento de Ciencias Políticas \\ UNED
}

\section{ELECCIONES Y PROCESO DE TRANSICIÓN EN CHILE}

La sucesión precipitada de cambios en las últimas semanas, verdadero vuelco de la Sociedad Internacional que hasta hoy nos habia tocado vivir, ha dejado en un segundo plano las elecciones presidenciales y legislativas en Chile, acontecimiento de transcendencia fundamental en el retorno de la democracia a este pais y en la desaparición de la última dictadura en América Latina.

La necesidad de una reflexión sincera de lo que han sido estas elecciones y de las «puertas" que se abren en el difícil y complejo proceso político chileno, mueven el interés de estas líneas con la seguridad de que los pasos necesarios que se han de dar en los próximos meses deben partir de un análisis crítico de los diferentes intereses de todo tipo representados por los distintos grupos políticos que han entrado en juego en esta liza electoral. Estudiar, en resumen, el abanico de posiciones y actitudes políticas, para saber con lo que se cuenta en el proceso de transición democrática.

En estas elecciones no se han enfrentado posiciones de partido con perfecta diferenciación ideológica y programática, lo que estaba en juego era el propio proceso de transición. Decidir, a fin de cuentas, entre la posibilidad de llevar a cabo el inicio de un verdadero proceso de transición a un sistema democrático o, por el contrario, dar paso a un sistema «híbrido" en donde la cara civil de la dictadura seguiria controlando directamente el poder político. 
Las opciones presidenciales que se presentaban al electorado eran: elegir un candidato como Patricio Aylwin, administrador de la victoria de las fuerźas políticas democráticas èn el plebistico de octubre, apoyado por la concertación de numerosas fuerzas políticas (desde la Democracia Cristiana hasta el Partido Comunista, pasando por el MIR) que se comprometía a llevar a cabo una transformación de la realidad política chilena desde la moderación cristiano-reformista, la negociáción y la reconciliación de todos los chilenos; o elegir una posición de cóntinuismo autoritario de presunto éxito económico que vaciaría de sentido y contenido la reforma, encarnadas en ell candidato Hernan Büchi.

Seria presuntuoso y fácil afirmar, ahora cuando ya se saben los resultados electorales, que la candidatura de Büchi estaba derrotada meses antes de llegar a los comicios, pero es ùn ejercicio necesario reflexionar sobre el camino de esta "derrota anunciada", conocer si era el candidato más adecuado e intuir cuál púede sér en un futuro la posición de la derecha económica y política continuista. Ésta sérá, sin duda, una de las piezas clave en el proceso de transición.

Hernán Büchi abandonó el gabinete como Ministro de Hacienda de Pinochet el 5 de abril pasado; desde ese momento hasta su presentación como futuro Presidente de Chile (brillantemente señalado por Genaro Arriaga en La Época,: '13 de diciembre) habría de estar condenàdo, no sólo a una contradicción política como depositario de la herencia pinochetista dentro de un medio electivo democrático; sino también envuelto en una contrariedad vital.

Empecemós por esta ủltima, la contrariedäd vital le llevó a renunciar en un primer momento a su nominación cuando ya aglutinaba a grandes sectores políticos y sociales confesando públicamente que no tenía vocación de candidato. Hasta ese momento, su esfuerzo estaba centrado en tomar distancia de la dictadura, así lo demuestran sus manifestaciones y también sus hechos, basta recordar su visita a la Vicaría de la Solidaridad, símbolo de la defensa de los derechos humanos:

Las presiones de Pinochet y de sectores económicos de gran peso le llevaron a la contradicción política y sólo un mes después anunciaba su candidatura aglutinando en su persona a las fuerzas que habían sido la columna vertebral del régimen en estos dieciséis años: Ia UDI (Unión Democrática Independiente), Renovación Nacional, los grandes grupos económicos, el gobierno, Pinochet y la mayor parte de las fuerzas armadas.

La vuelta de. Büchi y la constitución del pacto «Democracia y Progreso", tuvo en Pablo Baraona, Ministro de Economia en ese momento, el coordinador general de dicha operación, hombre de confianza de los grupos económicos, colaborador de Pinochet y militante de Renovación $\mathrm{Na}$ cional, el partido creado por Onofre Jarpa. Baraona asumió esta operación, antes incluso de que se formalizara la candidatura de Büchi, y debia lograr cinco objetivos: 
1. Obtener una sola candidatura en la derecha vinculada a la dictadura que aglutinara y ampliara el voto Sí del pasado plebiscito $(42 \%)$.

2. Garantizar la «ortodoxia» de la campaña, evitando cualquier desvío crítico hacia Pinochet (como visitas a la Vicaría o determinados acuerdos parlamentarios).

3. Confeccionar las listas propiciando el acuerdo dentro de los miembros del Pacto.

4. Conformar un programa de gobierno.

5. Canalizar y administrar los recursos provenientes del gobierno y del sector privado hacia Büchi.

Las primeras gestiones para componer las listas de candidatos a diputados fue un rotundo fracaso. No hubo una, sino cinco listas parlamentarias, y Baraona, que no gozaba de la confianza de los miembros del Pacto, ni tan siquiera de su propio partido, no pudo desde el gabinete poner orden en el caos que reinaba. Sólo obtuvo un logro: vincular a los cargos políticos de la dictadura a la nueva situación electoral. Si se observa la lista de RN-UDI, ésta era una interminable nómina de ex alcaldes, ex gobernadores y ex altos cargos del régimen; basta señalar que de 120 candidatos, 65 eran ex alcaldes.

La excusa aducida por Baraona para este significativo traspiés era que se habia llegado demasiado tarde a las negociaciones para propiciar la unidad de la derecha en el Pacto. Es cierto que los partidos de la Concertación para la Democracia habian logrado un año antes llegar a compromisos que propiciaron su victoria electoral en el plebiscito y partían de una base consensual más consolidada; no obstante, existieron otras cuestiones de mayor peso que son necesarias valorar:

El relanzamiento de Büchi como candidato supuso, después de una muy dura negociación, desandar el camino recorrido por Sergio Onofre Jarpa que, en ese momento, era el líder indiscutido de la derecha, cargado de honores por su destacada participación - junto con Aylwin y el ministro del Interior, Cáceres - en las negociaciones que condujeron a las reformas constitucionales votadas el 30 de julio. Jarpa acababa de ser nominado candidato presidencial por su partido y tendria que haber sido el hombre de la derecha en estas elecciones presidenciales para competir en términos de igualdad con Aylwin. Nunca un éxito político como su nominación fue más efímero, en la tarea de rechazo estuvo la UDI trabajando desde el principio y obtuvo que los apoyos económicos le fueran retirados. No conviene olvidar que Jarpa fue el primer político de la derecha que la misma 
noche del plebiscito de octubre se desmarcó del derrotado Pinochet para reconocer en los medios de comunicación que el dictador era un hombre políticamente acabado.

Finalmente se llegó a un acuerdo. En largas reuniones donde Baraona jugó un destacado papel, Jarpa renunció y Renovación Nacional cedió el apoyo a Büchi. Sin embargo, en el Comando que coordinaría la campaña del candidato existiría una gran mayoría de hombres de confianza del régimen con un gran predominio de la UDI y del Ilamado equipo económico de Chicago del cual Büchi era su mejor exponente.

En agosto la prensa nacional (Mercurio, 13 agosto. 1989) publicó un organigrama de la campaña de Büchi en donde se consagraba a Baraona como "generalísimo» de ésta, renunciando al ministerio pocos dias después. Lleno de pletórico entusiasmo, por un candidato que no era el líder natural de la derecha, Baraona caminó hacia el segundo gran fracaso de la campaña a su cargo: la conformación de las listas de senadores.

En esta cuestión tampoco hubo acuerdo, la situación continuó en proceso de abierta confrontación y hasta el punto de llegar al fracaso que el tiempo demostraría brutal: mientras la Concertación Democrática inscribia un candidato presidencial, la derecha inscribía a dos.

Al inicio, la soberbia llevó a minimizar el riesgo. Errázuriz, el otro candidato de la derecha continuista, empresario venido a más en el período de la dictadura con cierto prestigio en algunos sectores económicos e imagen de american-candidate, no tendria posibilidades. Luego se recurrió, por parte de Büchi, a un argumento falaz: «La candidatura de Errázuriz sólo restaría votos a Aylwin».

Otro aspecto que conviene valorar fue el tremendo esfuerzo para concertar un programa de gobierno para Büchi, este problema no se solventó y derivó en una lista de medidas, muchas de ellas de corte populista como la creación de un millón de puestos de trabajo, seguro de desempleo para todos, subida de todas las pensiones. Todo ello, sin hacer alusión al tipo de «sociedad democrática» que se quería construir: nada referido a sistema de derechos y libertades, ninguna referencia a la política exterior, ni a las fuerzas armadas, ni a la justicia y menos al régimen de garantías constitucionales.

A fines de septiembre la derecha parecía en bancarrota: habia una fragmentación de listas parlamentarias; no habia programa, los partidos que apoyaban a Büchi estaban excluidos de la campaña electoral por un «generalísimo» $y$, por si fuera poco, no había un candidato presidencial, sino dos con la candidatura de Errázuriz consolidada. Aun así, la campaña publicitaria y el marketing desplegados con apoyo económico considerable, la utilización de los medios de comunicación públicos y semi-públicos, salvarian la candidatura. 
EI CEP, institución ligada a la derecha pero de alta fiabilidad en ma-

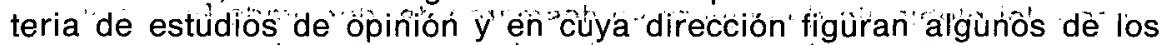

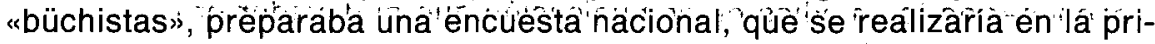
mera semana de octubre. La atención del comando Büchi se volcó en esta encuesta. Los resultados demostraban que desde marzo, Büchi apenas había subido un $0,2 \%$ en tanto Aylwin lo habia heçho en 3,3 puntos. Pero lo más grave era el análisis cualitativo del estudio: Büchi habia perdido tremendo atractivo como personá, en la apreciación de su capacidad para gobernar, su honradez y su capacidad para crear confianza. Por otro lado, la composición del voto Büchi había variado desfávorab́lémente, puès manteniéndose igual en el tótal hábía caído entré los sectorés jóvenes (mayoría electoral), siendo esa.disminución-compensada por grandes aumentos entre las personas de mayor edad ti:

A partir de ese momento la campaña de Büchi, entró en una fase de

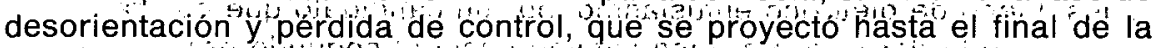
campaña. En la propaganda, las contradicciones se hicieron evidentes. Büchi en los últimós diás de campaña fue, à ratos, un técnicó respon'sable y, en otros momentos; un, populista de ofertas demagógicas que la economía no podía sostener Fue, un joven austero, respetuoso conisul oponente, para luego, bruscamente, aparecer como i un, candidato ,programado para una

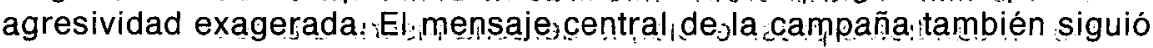
ese ritmo y paso de «Büchi es el hombre» a «Büchi es diferente», ejemplo

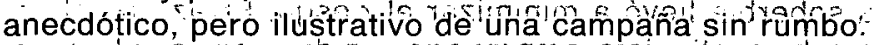

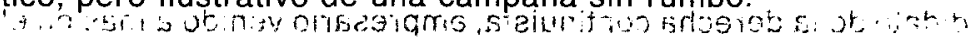

Las pugnas parlamentarias entre las distas de la, derecha hizo necesario la directa intervención de Pinochet que pidió, a los, 19 candidatos que no tenian una opción electoral real a renunciar a sus candidaturas en favor de Büchi. El desastre se veía llegar y el propio Jarpa se desligó de la campaña en un último momento: «No hemos tenido participación en la

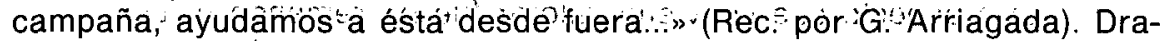
mático testimonio dé quien es el líder del únicó pártidó pólítico con peso

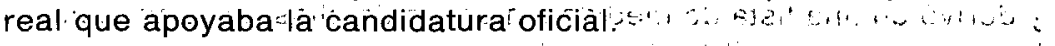

E É útimo recurso que restaba por utilizar para no hacer posible lo que parecia ya evidente, inténtaba poner al descubierto cómo detrás del acuerdo de todas las fuerzas democráticas no habia un acuérdo real sino un «pacto secreto en donde el Partido comunista era el eje de la futura política parlamentaria y de gobierno.

El resultado final de todo este proceso puede observarse en los resultadós electórales (Tabla 1$)$.

La:descripción crítica: realizada de la campaña de Büchi, no debe llevar a la conclusión de que la victoria del candidato de las fuerzas democráticas vino de la mano de tos errores del,contrario.

La campáña de Pátricio Aŷlwiñ siguiendò lás línès generales de la realizadá en èl plébiscito, fue capaz de superar grandes 'óbstáculos. Era 


\title{
ELECCIONES 1989
}

\author{
TABLA 1
}

CÓMPUTOS GENERALES

(Cifras del Ministerio del Interior con casi el 100 por 100 de los votos escrutados)

\begin{tabular}{|c|c|c|c|c|c|}
\hline \multicolumn{7}{|c|}{ ELECCION PRESIDENCIAL } \\
\hline TOTAL VOTOS & $\%$ & TOTAL VOTOS & $\%$ & TOTAL VOTOS & $\%$ \\
\hline 2.046 .580 & 29,39 & 1.074 .210 & 15,43 & 3.842 .887 & 55,2 \\
\hline \multicolumn{3}{|c|}{ HERNAN BÜCHI } & FCO. JAVIER ERRAZURIZ & \multicolumn{2}{|c|}{ PATRICIO AYLWIN } \\
\hline
\end{tabular}

\begin{tabular}{|c|c|c|c|c|c|c|c|c|c|c|c|c|c|}
\hline \multicolumn{14}{|c|}{ ELECCION PRESIDENGIAL POR REGIONES } \\
\hline & \multicolumn{2}{|c|}{ H. BÜCHI } & \multicolumn{2}{|c|}{ F. ERRAZURIZ } & \multicolumn{2}{|c|}{ P. AYLWIN } & \multirow[b]{2}{*}{$\begin{array}{c}\text { Total de } \\
\text { votos vallidos }\end{array}$} & \multirow[b]{2}{*}{ \% } & \multirow[b]{2}{*}{ Blancos } & \multirow[b]{2}{*}{ x } & \multirow[b]{2}{*}{ Nulos } & \multirow[b]{2}{*}{ * } & \multirow[b]{2}{*}{ Total de votos } \\
\hline REGION & VOTOS & x & votos & $x$ & vOTOS & * & & & & & & & \\
\hline I & 52.254 & 31,5 & 31.251 & 18,84 & 82.390 & 49,6 & 165.895 & 97,3 & 1.719 & 1,0 & 2.857 & 1,7 & 170.471 \\
\hline ॥ & 52.022 & 24,8 & 36.727 & 17,5 & 120.694 & 57,6 & 209.443 & 97,6 & 1.974 & 0,9 & 3.192 & 1,5 & 214.609 \\
\hline III & 33.863 & 30,3 & 10.094 & 9,0 & 67.803 & 59,1 & 111.760 & 97,5 & 1.499 & 1,3 & 1.300 & 1,2 & 114.649 \\
\hline IV & 75.725 & 30,7 & 29.984 & 12,1 & 141.114 & 57,2 & 246.823 & 97,0 & 3.874 & 1,5 & 3.895 & 1,5 & 254.692 \\
\hline v & 219.163 & 28,9 & 139.511 & 18,4 & 398.142 & 52,6 & 756.816 & 97,6 & 7.478 & 0,9 & 10.458 & 1,3 & 774.552 \\
\hline METROP. & 872.281 & 31,3 & 349.696 & 12,5 & 1.568 .227 & 56,2 & 2.790 .204 & 97,9 & 22.760 & 0,8 & 36.695 & 1,3 & 2.849 .660 \\
\hline VI & 109.126 & 29,6 & 43.077 & 11,7 & 215.876 & 58,6 & 368.079 & 97,3 & 5.003 & 1,3 & 5.177 & 1,4 & 378.259 \\
\hline VII & 125.698 & 28,5 & 63.956 & 14,5 & 251.788 & 57,0 & 441.442 & 97,1 & 5.811 & 1,3 & 7.172 & 1,6 & 454.425 \\
\hline VIII & 221.199 & 24,9 & 166.797 & 18,8 & 499.733 & 56,3 & 887.729 & 97,0 & 11.482 & 1,3 & 15.428 & 1,7 & 914.639 \\
\hline IX & 112.975 & 28,6 & 95.665 & 24,2 & 186.208 & 47,2 & 394.849 & 96,7 & 5.755 & 1,4 & 7.910 & 1,9 & 408.514 \\
\hline$x$ & 136.777 & 29,0 & 93.974 & 19,9 & 241.602 & 51,2 & 472.363 & 97,0 & 6.569 & 1,4 & 7.767 & 1,6 & 486.699 \\
\hline$X I$ & 11.556 & 31,0 & 5.320 & 14,3 & 20.392 & 54,7 & 37.268 & 97,3 & 510 & 1,3 & 519 & 1,4 & 38.297 \\
\hline XII & 23.941 & 29,6 & 8.157 & 10,1 & 48.908 & 60,4 & 81.006 & 98,2 & 635 & 0,8 & 873 & 1,1 & 82.514 \\
\hline
\end{tabular}

(Fuente: La Época, 10-XII-89). 


\begin{tabular}{|c|c|c|}
\hline \multicolumn{3}{|c|}{$\begin{array}{c}\text { CIRCUNSCRIPCIÓN } 1 \\
\text { I Región de Tarapaca Distritos } 1 \text { y } 2\end{array}$} \\
\hline Pacto & $\begin{array}{l}\text { Total } \\
\text { votos }\end{array}$ & $\%$ \\
\hline \multirow{3}{*}{$\begin{array}{l}\text { CONCERTACION } \\
\text { Humberto Palza } \\
\text { (DC) } \\
\text { Anibal Palma (Ind.) }\end{array}$} & 76.208 & 44,7 \\
\hline & 45.326 & 26,6 \\
\hline & 30.885 & 18,1 \\
\hline \multirow{3}{*}{$\begin{array}{l}\text { RN-UDI } \\
\text { Gabriel Abusie- } \\
\text { me (RN) } \\
\text { Julio Lagos (Ind.) }\end{array}$} & 49.551 & 29,1 \\
\hline & 7.471 & 4,4 \\
\hline & 42.080 & 24,7 \\
\hline \multirow{3}{*}{$\begin{array}{l}\text { PL-PSCH } \\
\text { Julio Barrraquel } \\
\text { (Ind.) } \\
\text { Raquel Pino (Ind.) }\end{array}$} & 6.317 & 3,7 \\
\hline & 2.143 & 1,3 \\
\hline & 2.174 & 2,5 \\
\hline \multicolumn{3}{|l|}{ INDEPENDIENTES } \\
\hline Jorge Soria & 27.053 & 15,9 \\
\hline $\begin{array}{l}\text { Fernando Doug- } \\
\text { nac }\end{array}$ & 2.638 & 1,6 \\
\hline
\end{tabular}

\begin{tabular}{|c|c|c|}
\hline \multicolumn{3}{|c|}{$\begin{array}{l}\text { CIRCUNSCRIPCIÓN } 3 \\
\text { III Región de Atacama Distritos } \\
5 \text { y } 6\end{array}$} \\
\hline Pacto & $\begin{array}{l}\text { Toial } \\
\text { votos }\end{array}$ & $\%$ \\
\hline CONCERTACIÓN & 67.228 & 58,7 \\
\hline $\begin{array}{l}\text { Adolfo Zaldivar } \\
\text { (DC) }\end{array}$ & 30.933 & 27,0 \\
\hline $\begin{array}{l}\text { Ricardo Núñez } \\
\text { (PPD) }\end{array}$ & 36.295 & 31,7 \\
\hline \multirow{3}{*}{$\begin{array}{l}\text { RN-UDi } \\
\text { Ignacio Pérez Wal- } \\
\text { ker (RN) } \\
\text { Jonás Gómez (Ind.) }\end{array}$} & 36.282 & 31,7 \\
\hline & 24.150 & 21,1 \\
\hline & 12.132 & 10,6 \\
\hline \multirow{2}{*}{$\begin{array}{l}\text { PL-PSCH } \\
\text { Luis Bogdahic (PL) }\end{array}$} & 2.669 & 2,3 \\
\hline & 2.669 & 2,3 \\
\hline \multirow{2}{*}{$\begin{array}{l}\text { INDEPENDIENTE } \\
\text { Elías Nehme }\end{array}$} & 2.572 & 2,3 \\
\hline & 2.572 & 2,2 \\
\hline
\end{tabular}

\begin{tabular}{|l|c|c|}
\hline \multicolumn{3}{|c|}{ CIRCUNSCRIPCION 2} \\
\multicolumn{2}{|c|}{ II Región de Antofagasta Distritos } \\
3 y 4 \\
\hline \multicolumn{1}{|c|}{ Pacto } & $\begin{array}{c}\text { Total } \\
\text { votos }\end{array}$ & $\%$ \\
\hline ConcERTACIon & 84.409 & 39,3 \\
\cline { 2 - 3 } $\begin{array}{l}\text { Carmen Frei (DC) } \\
\text { Bernardo Julio (PR) }\end{array}$ & 58.814 & 27,4 \\
\cline { 2 - 3 } & 25.595 & 11,9 \\
\hline RN-udI & 71.036 & 33,1 \\
\cline { 2 - 3 } $\begin{array}{l}\text { Radozlav Razmi- } \\
\text { lic (RN) }\end{array}$ & 29.368 & 13,7 \\
\cline { 2 - 3 } $\begin{array}{l}\text { Arturo Alessandri } \\
\text { (Ind.) }\end{array}$ & 41.668 & 19,4 \\
\hline PAIS-PRsD & 48.596 & 22,7 \\
\cline { 2 - 3 } $\begin{array}{l}\text { Fanny Pollarolo } \\
\text { (PAIS) }\end{array}$ & 46.601 & 21,7 \\
\cline { 2 - 3 } $\begin{array}{l}\text { Gustavo Araya } \\
\text { (PAIS) }\end{array}$ & 1.995 & 0,9 \\
\hline
\end{tabular}

\begin{tabular}{|l|c|c|}
\hline \multicolumn{3}{|c|}{ CIRCUNSCRIPcIó 4} \\
\multicolumn{2}{|c|}{ IV Región de Coquimbo Distritos } \\
\multicolumn{2}{|c|}{ Pacto 9}
\end{tabular}




\begin{tabular}{|c|c|c|}
\hline \multicolumn{3}{|c|}{$\begin{array}{c}\text { CIRCUNSCRIPción } 5 \\
\text { V interior, } V \text { Región de Valpo. Dis- } \\
\text { tritos } 10,11 \text { y } 12\end{array}$} \\
\hline Pacto & $\begin{array}{l}\text { Total } \\
\text { votos }\end{array}$ & $\%$ \\
\hline \multirow{3}{*}{$\begin{array}{l}\text { CONCERTACION } \\
\text { Carlos González } \\
\text { (PR) } \\
\text { Orlando Sáenz } \\
\text { (Ind.) }\end{array}$} & 131.951 & 36,5 \\
\hline & 72.736 & 20,1 \\
\hline & 59.215 & 16,4 \\
\hline \multirow{3}{*}{$\begin{array}{l}\text { RN-UDI } \\
\text { Sergio Romero } \\
\text { (Ind.) } \\
\text { Edmundo Elu- } \\
\text { chans (Ind.) } \\
\end{array}$} & 121.706 & 33,6 \\
\hline & 71.562 & 19,8 \\
\hline & 50.144 & 13,9 \\
\hline \multirow{3}{*}{$\begin{array}{l}\text { PL-PSCH } \\
\text { Sergio Valencia } \\
\text { (Ind.) } \\
\text { Raúl Silva (Ind.) } \\
\end{array}$} & 24.387 & 6,7 \\
\hline & 11.766 & 3,3 \\
\hline & 12.621 & 3,5 \\
\hline \multirow{3}{*}{$\begin{array}{l}\text { PAIS-PRSD } \\
\text { Luis Guastavino } \\
\text { (PAIS) } \\
\text { Jose Freire (PAIS) }\end{array}$} & 62.975 & 17,4 \\
\hline & 60.600 & 16,7 \\
\hline & 2.375 & 0,7 \\
\hline
\end{tabular}

\begin{tabular}{|c|c|c|}
\hline \multicolumn{3}{|c|}{$\begin{array}{c}\text { Circunscripción } 6 \\
\text { V Costa, V Región de Valpo. Distri- } \\
\text { tos } 13,14 \text { y } 15\end{array}$} \\
\hline Pacto & $\begin{array}{l}\text { Total } \\
\text { votos }\end{array}$ & $\%$ \\
\hline CONCERTACIÓN & 226.963 & 55,0 \\
\hline Juan Hamilton (DC) & 112.626 & 27,3 \\
\hline Laura Soto (PPD) & 114.337 & 27,7 \\
\hline RN-UDI & 137.335 & 33,3 \\
\hline $\begin{array}{l}\text { Gonzalo Yussel } \\
\text { (RN) }\end{array}$ & 68.127 & 16,5 \\
\hline $\begin{array}{l}\text { Beltrán Urenda } \\
\text { (Ind.) }\end{array}$ & 69.208 & 16,8 \\
\hline
\end{tabular}

\section{CIRCUNSCRIPCION 7}

Región Metropolitana Poniente Distrit. $16,17,18,19,20,22,30$ y 31

\begin{tabular}{|l|c|c|}
\hline \multicolumn{1}{|c|}{ Paclo } & $\begin{array}{c}\text { Total } \\
\text { votos }\end{array}$ & $\%$ \\
\hline CONCERTACIón & 807.298 & 58,9 \\
\cline { 2 - 3 } $\begin{array}{l}\text { Andrés Zaldivar } \\
\text { (DC) }\end{array}$ & 407.890 & 29,8 \\
\cline { 2 - 3 } $\begin{array}{l}\text { Ricardo Lagos } \\
\text { (PPD) }\end{array}$ & 399.408 & 29,2 \\
\hline $\begin{array}{l}\text { RN-uDI } \\
\text { Miguel Otero (RN) }\end{array}$ & 423.905 & 30,9 \\
\cline { 2 - 3 } $\begin{array}{l}\text { Jaime Guzmán } \\
\text { (UDI) }\end{array}$ & 199.603 & 14,6 \\
\hline PL-Psch & 224.302 & 16,4 \\
\hline $\begin{array}{l}\text { Sergio Santander } \\
\text { (Ind.) }\end{array}$ & 73.209 & 5,3 \\
\cline { 2 - 3 } $\begin{array}{l}\text { Rodrigo Miranda } \\
\text { (Ind.) }\end{array}$ & $\mathbf{1 3 . 4 1 4}$ & 1,0 \\
\hline
\end{tabular}

CIRCUNSCRIPCIÓN 8

Región Metrop. Oriente

Distrit. $21,23,24,25,26,27,28$ y 29

\begin{tabular}{|l|c|c|}
\hline \multicolumn{1}{|c|}{ Pacto } & $\begin{array}{c}\text { Total } \\
\text { votos }\end{array}$ & $\%$ \\
\hline ConCERTACión & 822.531 & 56,0 \\
\cline { 2 - 3 } $\begin{array}{l}\text { Eduardo Frei (DC) } \\
\text { Maria E. Carrera } \\
\text { (Ind.) }\end{array}$ & 603.091 & 41,1 \\
\hline RN-udı & 219.440 & 15,0 \\
\hline $\begin{array}{l}\text { Sebastían Piñera } \\
\text { (Ind.) }\end{array}$ & 527.073 & 35,9 \\
\cline { 2 - 3 } $\begin{array}{l}\text { Hermógenes Pé- } \\
\text { rez de A. (Ind.) }\end{array}$ & 206.515 & 14,1 \\
\hline PL-PScH & 42.254 & 2,9 \\
\cline { 2 - 3 } Sergio Vial (Ind.) & 42.254 & 2,9 \\
\hline PN & 22.634 & 1,5 \\
\cline { 2 - 3 } Carmen Sáenz (PN) & 22.634 & 1,5 \\
\hline
\end{tabular}




\begin{tabular}{|c|c|c|}
\hline $\begin{array}{r}\text { CIRcunsc } \\
\text { VI Región del } \\
\text { Distritos } 32 \\
\end{array}$ & $\begin{array}{l}\text { PCION } 9 \\
\text { B. O'Hig } \\
3,34 \text { y } 3\end{array}$ & \\
\hline Pacto & $\begin{array}{l}\text { Total } \\
\text { votos }\end{array}$ & $\%$ \\
\hline CONCERTACION & 212.005 & 56,0 \\
\hline Nicolás Diaz (DC) & 106.062 & 28,0 \\
\hline Anselmo Sule (Ind.) & 105.943 & 28,0 \\
\hline RN-UDI & 98.467 & 26,0 \\
\hline Alfonso Oruela (RN) & 62.823 & 16,6 \\
\hline $\begin{array}{l}\text { Manuel Valdés } \\
\text { (Ind.) }\end{array}$ & 35.644 & 9,4 \\
\hline AN-DR & 40.319 & 10,7 \\
\hline $\begin{array}{l}\text { Domingo Durán } \\
\text { (DR) }\end{array}$ & 15.264 & 4,0 \\
\hline $\begin{array}{l}\text { Rafael Cumsille } \\
\text { (Ind.) }\end{array}$ & 25.055 & 6,6 \\
\hline PL-PSCH & 7.221 & 1,9 \\
\hline Ricardo Bustos (PL) & 7.221 & 1,9 \\
\hline
\end{tabular}

\begin{tabular}{|c|c|c|}
\hline \multicolumn{3}{|c|}{$\begin{array}{c}\text { CIACUNSCAIPCION } 11 \\
\text { VII Sur, VII Región del Maule } \\
\text { Distritos } 39 \text {, y } 40\end{array}$} \\
\hline Pacto & $\begin{array}{l}\text { Total } \\
\text { votos }\end{array}$ & $\%$ \\
\hline CONCERTACION & 84.345 & 50,0 \\
\hline $\begin{array}{l}\text { José Tomás Sáenz } \\
\text { (PH) }\end{array}$ & 35.575 & 21,1 \\
\hline Mario Papi (Ind.) & 48.770 & 28,9 \\
\hline RN-UDI & 64.661 & 38,4 \\
\hline $\begin{array}{l}\text { Sergio Onofre Jar- } \\
\text { pa (RN) }\end{array}$ & 55.807 & 33,1 \\
\hline $\begin{array}{l}\text { Rolando Renteria } \\
\text { (RN) }\end{array}$ & 8.854 & 5,3 \\
\hline PL-PSCH & 9.764 & 5,8 \\
\hline $\begin{array}{l}\text { Aldo Roncaglio } \\
\text { (Ind.) }\end{array}$ & 6.811 & 4,0 \\
\hline $\begin{array}{l}\text { Atiliano Parada } \\
\text { (Ind.) }\end{array}$ & 2.953 & 1,8 \\
\hline
\end{tabular}

\begin{tabular}{|c|c|c|}
\hline \multicolumn{3}{|c|}{$\begin{array}{c}\text { CIRCUNSCRIPCION } 10 \\
\text { VII Norte, VII Región del Maule } \\
\text { Distritos } 36,37, \text { y } 38 \\
\end{array}$} \\
\hline Pacto & $\begin{array}{l}\text { Total } \\
\text { votos }\end{array}$ & $\%$ \\
\hline CONCERTACION & 171.167 & 59,9 \\
\hline $\begin{array}{l}\text { Jaime Gazmuri } \\
\text { (PPD) }\end{array}$ & 56.931 & 19,9 \\
\hline $\begin{array}{l}\text { Máximo Pacheco } \\
\text { (DC) }\end{array}$ & 114.236 & 40,0 \\
\hline RN-UDt & 82.027 & 28,7 \\
\hline $\begin{array}{l}\text { Alberto Cardemil } \\
\text { (RN) }\end{array}$ & 66.353 & 23,2 \\
\hline $\begin{array}{l}\text { Silvio Rodríguez } \\
\text { (Ind.) }\end{array}$ & 15.674 & 5,5 \\
\hline PL-PSCH & 16.692 & 5,8 \\
\hline $\begin{array}{l}\text { Guido Briceño } \\
\text { (PSCH) }\end{array}$ & 4.257 & 1,5 \\
\hline Patricio Parol (Ind.) & 12.435 & 4,4 \\
\hline
\end{tabular}

\section{CIRCUNSCRIPCION 12}

VIII Norte, VIII Región del Bio-Bio Distritos $42,43,44$, y 45

\begin{tabular}{|l|c|c|}
\hline \multicolumn{1}{|c|}{ Pacto } & $\begin{array}{c}\text { Total } \\
\text { votos }\end{array}$ & $\%$ \\
\hline ConCERTACIÓN & 195.816 & 36,2 \\
\cline { 2 - 3 } Arturo Frei (DC) & 195.816 & 36,2 \\
\hline \multirow{2}{*}{$\begin{array}{l}\text { RN-UDI } \\
\text { Renato Gazmuri } \\
\text { (RN) }\end{array}$} & 157.940 & 29,2 \\
\cline { 2 - 3 } $\begin{array}{l}\text { Eugenio Cantua- } \\
\text { rias (UDI) }\end{array}$ & 74.245 & 13,7 \\
\hline AN-DR & 83.695 & 15,5 \\
\hline Fidel Reyes (Ind.) & 34.972 & 6,5 \\
\hline PAIS-PRSD & 124.908 & 23,1 \\
\cline { 2 - 3 } \begin{tabular}{l} 
Luis Maira (PAIS) \\
\cline { 2 - 3 }
\end{tabular} & 124.908 & 23,1 \\
\hline
\end{tabular}




\begin{tabular}{|c|c|c|}
\hline \multicolumn{3}{|c|}{$\begin{array}{l}\text { CIRCUNSCRIPCION } 13 \\
\text { VIII Sur, VIII Región del Bio-Bio } \\
\text { Distritos } 41,46 \text { y } 47\end{array}$} \\
\hline Pacto & $\begin{array}{l}\text { Total } \\
\text { votos }\end{array}$ & $\%$ \\
\hline \multirow{3}{*}{$\begin{array}{l}\text { CONCERTACIón } \\
\text { Edgardo Condeza } \\
\text { (PPD) } \\
\text { Mariano Ruiz-Es- } \\
\text { quide (DC) }\end{array}$} & 207.064 & 55,4 \\
\hline & 98.566 & 26,4 \\
\hline & 108.498 & 29,0 \\
\hline \multirow{3}{*}{$\begin{array}{l}\text { RN-UDI } \\
\text { Mario Ríos (RN) } \\
\text { Guillermo Arthur } \\
\text { (Ind.) }\end{array}$} & 141.281 & 37,8 \\
\hline & 72.123 & 19,3 \\
\hline & 69.158 & 18,5 \\
\hline
\end{tabular}

\begin{tabular}{|c|c|c|}
\hline \multicolumn{3}{|c|}{$\begin{array}{c}\text { CIRCUNSCRIPCIÓN } 15 \\
\text { IX Sur, Región de La Araucania } \\
\text { Distritos } 50,51, \text { y } 52\end{array}$} \\
\hline Pacto & $\begin{array}{l}\text { Total } \\
\text { votos }\end{array}$ & $\%$ \\
\hline \multirow{3}{*}{$\begin{array}{l}\text { CONCERTACIÓN } \\
\text { Jorge Lavandero } \\
\text { (DC) } \\
\text { Julio Subercaseaux } \\
\text { (PPD) }\end{array}$} & 121.930 & 46,8 \\
\hline & 106.883 & 41,0 \\
\hline & 15.047 & 5,8 \\
\hline \multirow{3}{*}{$\begin{array}{l}\text { RN-UDI } \\
\text { Sergio Diez (Ind.) } \\
\text { Víctor Carmine (RN) }\end{array}$} & 79.532 & 30,5 \\
\hline & 53.577 & 20,6 \\
\hline & 25.955 & 9,9 \\
\hline \multirow{2}{*}{$\begin{array}{l}\text { P. SuR } \\
\text { Eduardo Diaz } \\
\text { (P. Sur) }\end{array}$} & 45.276 & 17,4 \\
\hline & 45.276 & 17,4 \\
\hline
\end{tabular}

\begin{tabular}{|c|c|c|}
\hline \multicolumn{3}{|c|}{$\begin{array}{c}\text { CIRCunscripción } 14 \\
\text { IX Norte, Región de La Araucanía } \\
\text { Distritos } 48 \text { y } 49\end{array}$} \\
\hline Pacto & $\begin{array}{l}\text { Total } \\
\text { votos }\end{array}$ & $\%$ \\
\hline \multirow{3}{*}{$\begin{array}{l}\text { CONCERTACION } \\
\text { Erick Schnake } \\
\text { (PPD) } \\
\text { Ricardo Navarre- } \\
\text { te (PR) }\end{array}$} & 69.861 & 47,7 \\
\hline & 30.380 & 20,7 \\
\hline & 39.481 & 27,0 \\
\hline \multirow{3}{*}{$\begin{array}{l}\text { RN-UDI } \\
\text { Fernando Matura- } \\
\text { na (RN) } \\
\text { Francisco Prat (RN) }\end{array}$} & 46.157 & 31,5 \\
\hline & 16.549 & 11,3 \\
\hline & 29.608 & 20,2 \\
\hline \multirow{3}{*}{$\begin{array}{l}\text { PN } \\
\text { Santiago Garcia } \\
\text { (PN) } \\
\text { Patricio Phillips } \\
\text { (PN) }\end{array}$} & 20.973 & 14,3 \\
\hline & 3.688 & 2,5 \\
\hline & 17.285 & 11,8 \\
\hline
\end{tabular}

\begin{tabular}{|c|c|c|}
\hline \multicolumn{3}{|c|}{$\begin{array}{c}\text { CIRCUNSCRIPCION } 16 \\
\text { X Norte, Región de Los Lagos } \\
\text { Distritos } 53,54 \text {, y } 55\end{array}$} \\
\hline Pacto & $\begin{array}{l}\text { Total } \\
\text { votos }\end{array}$ & $\%$ \\
\hline \multirow{3}{*}{$\begin{array}{l}\text { CONCERTACIÓN } \\
\text { Gabriel Valdés (DC) } \\
\text { Francisco Rivas } \\
\text { (Ind.) }\end{array}$} & 143.368 & 55,5 \\
\hline & 102.782 & 39,8 \\
\hline & 40.586 & 15,7 \\
\hline \multirow{3}{*}{$\begin{array}{l}\text { RN-UDI } \\
\text { Enrique Larre (Ind.) } \\
\text { Agustín Acuña }\end{array}$} & 101.446 & 39,2 \\
\hline & 78.704 & 30,5 \\
\hline & 22.742 & 8,8 \\
\hline
\end{tabular}


fundamental desplegar los esfuerzos consensuados suficientes para seguir manteniendo la principal arma que habia propiciado la victoria en el plebiscito frente a Pinochet: la unidad.

Esta idea era común a todas las fuerzas progresistas. Todas eran conscientes de que el menos malo de los procesos de transición pasaba por este requisito, pero la búsqueda de un candidato presidencial único y el acuerdo de un programa consensuado de gobierno, exigía grandes sacrificios. Entre ellos, olvidar el propio origen de la dictadura. A nadie se le oculta que obtener el apoyo del Partido Socialista, Comunista y del MIR para un candidato demócratacristiano, no era labor fácil.

El pragmatismo de las cúpulas dirigentes reconociendo que era el único camino posible para no perder el proceso de transición y la madurez política de las bases, hicieron realidad lo que parecía imposible. La entrada en la DC de jóvenes dirigentes propugnando la defensa de los derechos humanos (Movimiento Sebastián Azevedo, Vicaría de Santiago), la presencia militante y el apoyo social que tiene en los barrios de mayor pobreza, su trabajo en el plebiscito y su oposición a Pinochet; todo ello llevó a crear la confianza en el resto de las fuerzas politicas de que Aylwin no estaba dispuesto a sacrificar la democracia por una segunda vez.

La Democracia Cristiana, por otra parte, era consciente de que una vez ganado el plebiscito en la nueva situación política, el protagonismo en las negociaciones con la Junta y con la derecha, pasaba por un candidato presidencial que obtuviera el respaldo y el acuerdo del resto de las fuerzas políticas democráticas. Patricio Aylwin tenía mucho camino recorrido porque su labor como portavoz del Comando del NO, le había llevado a un reconocimiento como interlocutor válido por parte oficialista y era un hombre respetado en los sectores de la izquierda.

El consenso obtenido por Aylwin con el resto de las fuerzas democráticas para introducir las reformas constitucionales necesarias que permitieran la participación de todos los partidos, independientemente de su ideología, le había llevado incluso a enfrentamientos con sectores de su propio partido que defendian un acuerdo más restringido. Las negociaciones que llevó a cabo con Cáceres y Jarpa estuvieron en todo momento sometidas al control y el consenso de las fuerzas políticas que le habian prestado su apoyo por encima de presiones de su partido.

La imagen de hombre conciliador y de compromiso, de sólida madurez política, estaba creada y sobre ésta se desarrollaria la campaña. El Presidente propugnado sería el símbolo de la reconciliación de todos los chilenos, que daba muestras de mesura y prudencia en sus juicios, con una visión política en la cual prima la solidez de los principios, unida a la sobriedad y ponderación en las actitudes y expresiones. La preocupación del candidato no era sólo ganar las elecciones, sino también ir preparando algunas de las cuestiones vitales en el futuro proceso de transición: Acuerdo económico (Banco Central), apoyo sindical, negociación empresarios- 
sindicatos, incorporación de sectores independientes, política exterior, etcétera, etc.

Dos reflexiones sobre la campaña presidencial, una referida a los aciertos en los pasos previos: la visita de Aylwin a los países europeos y los contactos realizados con los presidentes del área (criticados por ciertos sectores), se realizaron desde una visión de Estado que daba solidez a una campaña preocupada por la necesidad de recabar apoyos políticos y económicos al proceso de transición que se iniciaba con la elección del candidato de las fuerzas democráticas. Otra característica destacable se refiere al tono moderado de sus intervenciones que, como se vio en los resultados electorales, era una tendencia de voto mayoritaria en el país. Junto al discurso moderado y populista, la valentía de incorporar al proceso a los sectores más a la izquierda del panorama político y más concretamente al PAIS (Partido Sócialista de Almeyda, Izquierda Cristiana, Partido Comunista, MIR), aun con el riesgo que ello suponía de dar argumentos «descalificadores" a los partidos de la derecha que los utilizáron hasta la saciedad en toda la campaña. Un proceso de transición sin la incorporación de estos sectores húbiera sido necesariamente un proceso condenado al fracaso.

Aylwin fue el factor aglutinador y lo más fácil en toda la campaña; sin embargo, las elecçiones parlamentärias era una de las piezas angulares para poner'en marcha un auténtico proceso de transición a la democracia. Este procesó, necesariamente, debía y debe plantearse la reforma de la actual Constitución y de las principales leyés orgánicas, siendo requisito légal, para ello, el voto de la máyoria de dos terciós de las Cámaras.

Las elecciones de senadores y diputados se presentaban mucho más complicadas y dificiles; en ellas, los candidatos de los diferentes partidos políticos debian medir sus fuerzas bajo un marco jurídico èlectoral creado por la dictadura como uno de los "eslabones de oro" para controlar el proceso de transición. L'a posición de los partidos que ápoyaban a Aylwin en la campaña fúe desigual no sólo si lo comparamos con los dos partidos de la derecha (UDI y RN) sino incluso entre ellos mismos. La Democracia Cristiana gozaba no śólo de mayor apoyo económico, sino también de las estructuras políticas menos castigadás durante la dictàdura y había gozado de más tiempo para su acomodación a la nueva realidad politica, mientras la semi-legalidad de la izquierda y la división del Partido Socialista situaba a estos sectores en inferioridad'en el proceso electoral y también, como veremos después, en el proceso de transición.

Aun teniendo en cuenta los imponderables históricos a los que anteriormente nos hemos referido, el PPD (Partido para la Democracia) y el PAIS (Partido Amplio de izquierda Socialista), hicieron posible la elección de un presidente democrático, pero cometieron errores vitales en la campaña y en el proceso anterior de negociación para reformar las leyes cons- 
titucionales. Sobre los errores de la campaña señalar la poca coordinación existente entre los candidatos a ambas Cámaras de la izquierda (especialmente del PPD). Existió un reforzamiento de los candidatos más débiles por parte de los que parecia segura su elección y un exceso de confianza en los que se creia seguros. Este fue el caso de Ricardo Lagos, líder del PPD y figura destacada del socialismo chileno.

Los errores señalados, no obstante, son mínimos en comparación con el que señalábamos con anterioridad referido a la izquierda y a la reforma de las leyes orgánicas constitucionales. En el proceso de negociación llevado a cabo por Aylwin con los representantes de la Junta y la derecha, existió una despreocupación absoluta en lo que se refiere a la ley electoral. En las conversaciones de Aylwin con la izquierda como portavoz del pacto democrático, apareció como una de las cuestiones de segundo orden. Una ley electoral que potenciaba a los candidatos más votados de ambos bloques, y partiendo de un análisis realista de la situación política chilena era presumible que beneficiaria en mayor medida en el Pacto de la Concertación a la Democracia Cristiana que al PPD.

El sistema electoral, creado por Guzmán, asesinado hacía pocas semanas por un comando terrorista, presidente de la UDI y redactor de la actual Constitución, no era ni proporcional ni mayoritario, no tenía que ver con ninguno de los existentes en la actualidad y podría describirse como un sistema de "doble o nada". De acuerdo con la ley en cuestión, el artículo segundo señala lo siguiente: «Elegirá dos cargos aquella lista o nómina que tuviera dos candidatos y cuyo total de votos excediera el doble de los que alcanzare la lista que le sigue en número de sufragios. Si ninguna lista o nómina eligiera los dos cargos, elegirán un cargo cada una de las listas que obtengan las dos más altas mayorias en cada nómina..."

El resultado final de esta ley electoral nos lleva necesariamente a reflexionar sobre las serias dificultades que va a encontrar el proceso de transición. La primera es que el Congreso elegido no será el reflejo del equilibrio político real que existe en el país. Nueve senadores y quince diputados que hubieran correspondido a las fuerzas democráticas pasaron a las continuistas de derecha. Se quedaron fuera figuras imprescindibles para el proceso de transición como los presidentes de las coaliciones más importantes de la izquierda, el PAIS y el PPD, Luis Maira y Ricardo Lagos (este último ocupará el Ministerio de Educación del primer Gobierno democrático), tampoco existirá representación comunista en el Congreso. Las fuerzas democráticas pierden la mayoría en el Senado con los ocho senadores designados, cuatro de ellos por el Consejo de Seguridad y el resto fieles a Pinochet, como Sergio Fernández o William Thayer: El equilibrio en las Cámaras puede verse en la Tabla 2.

Con este equilibrio parlamentario de fuerzas, se supone difícil las reformas constitucionales y legales para desatar los nudos realizados por la dictadura al proceso de transición. Las próximas Cámaras deberán ad- 
ELECCIONES 1989

TABLA 2

COMPOSICIÓN DE LAS CÁMARAS
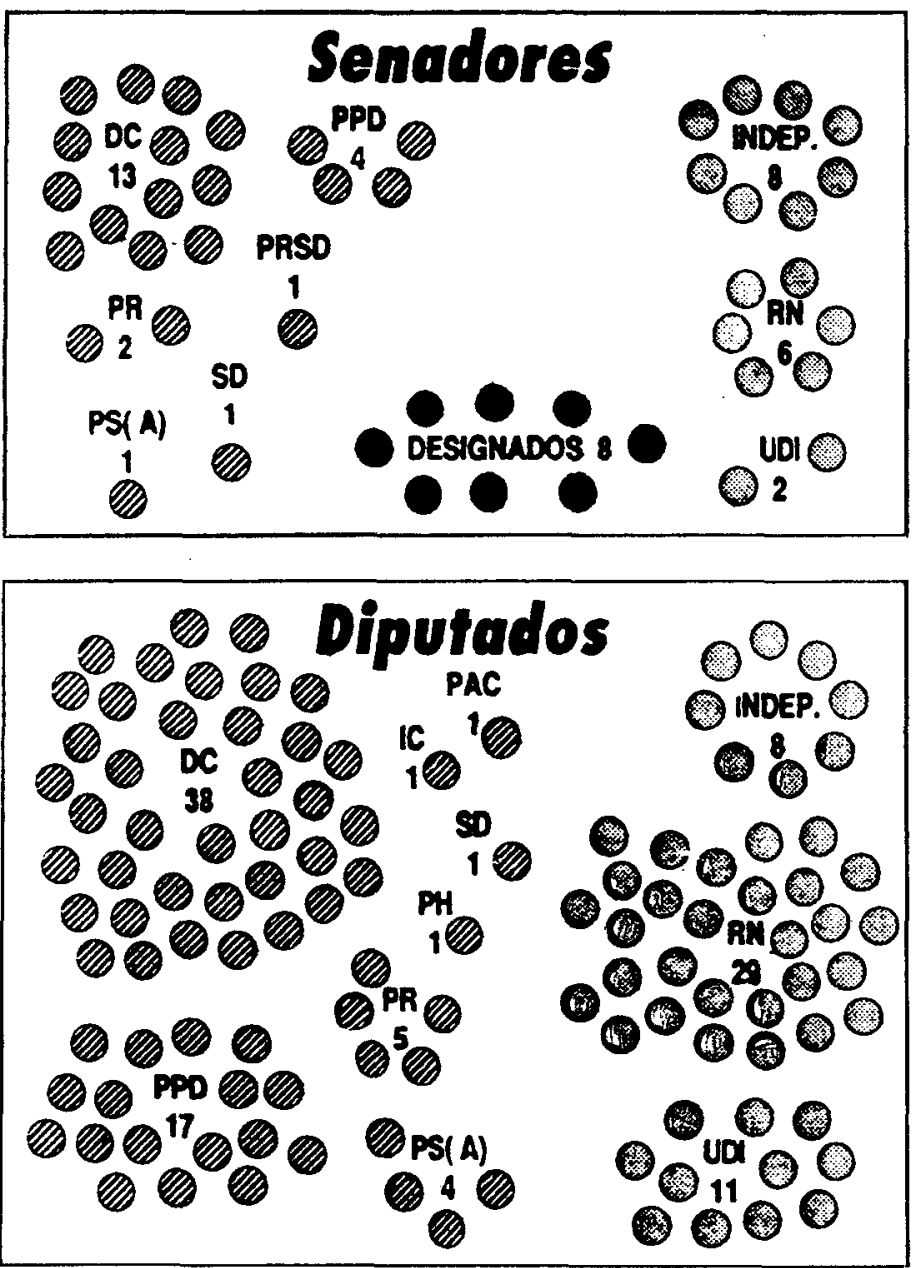

(Fuente: La Época, 10-XII-89). 
ministrar una Constitución de espíritu y sentido antidemocrático y, por lo tanto, necesariamente, deberán ser constituyentes, no en el sentido clásico del concepto - una de las peculiaridádè del proceso chileno en comparación con el uruguayo, argentino o el español es que su Constitución no es fruto del proceso de transición- pero sí en la práctica del día a dia de la labor legislativa.

Muchas y complejas tendrán que ser las reformas, más aún si se tiene en cuenta las limitaciones que tendrá el próximo Presidente de la República en el desarrollo del poder ejecútivo o, dicho de otra forma, muchos serán los obstáculos si se tiene en cuenta la red de poder legal del general Pinochet; ahora-y. luego, desde-su-futuro cargo de Presidente del Consejo de Seguridad Nacional. Éstos serán algunos de los ámbitos donde Pinochet, legalmente; tiene algo que decir:

- Ley de lás Fuerzas Armadas y Carabineros: Öbligáción consultiva iy decisoria. Inamovilidad del comandante en jefe Traspaso o sustitución de los miembros del Conséjó. Fin o ódesaparición de órganos militares (CNI).

— Ley Elèctoral: Senadores designados.

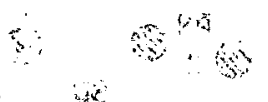

— Ley del Congreso: Imposibilidád para fiscâlizar actos de Gobiernos anteriores.

- Ley de Autonomía y Consejo del Banco Central: Obligación consultiva.

— Ley de Codelco y Corporación Chilena del Cobre: Óbligación consultiva.

- Ley de Privatizaciones: Obligación consultiva

- Ley del Póder Judicial: Obligación consuiltiva en las designaciones de jueces de la Corte Suprema o en la eliminación de los integrantes:

- Ley de inamovilidad de altos cargos de la Administración Pública: Obligación consultiva para el nuevo Estatuto Administrativo.

Los resultados electorales impedirán a la Concertación de Partidos por la Democracia, que obtuvo 22 senadores y 69 diputados, modificar o derogar por si sola ninguna de las más importantes leyes que heredará del actual régimen. Los tipos de quorum que se necesitan en el Parlamento 
para realizar las modificaciones legales, teniendo en cuenta las facultades consultivas de Pinochet, son los siguientes:

- Leyes de quorum calificado: Requieren la mayoria simple, es decir, 61 diputados y 23 senadores. Faltaria un senador. Aquí se incluyen las leyes sobre el Consejo Nacional de Televisión, sobre conductas terroristas y sobre el Estado-empresario.

- Leyes Orgánicas Constitucionales: Requieren del acuerdo de $4 / 7$ de los parlamentarios (68 diputados y 27 senadores). Faltarian cinco senadores. Aqui se encuentran la Ley del Banco Central, la Ley sobre las Fuerzas Armadas, la Ley Electoral: votaciones y escrutinio, la Ley de votación para municipalidades.

- Reformas a la Constitución: Se exige una mayoría de $3 / 5$ de los parlamentarios. Es decir, 72 diputados y 27 senadores. Faltarian tres diputados y cinco senadores.

- Reformas de las Bases Institucionales: Para modificar estas materias se requiere la aprobación de al menos 2/3 de los parlamentarios, esto es, 80 diputados y 31 senadores. Faltarían 11 diputados y nueve senadores. Esta exigencia es necesaria para modificar la Ley de Bases de la Institucionalidad, la Ley del Tribunal Constitucional, los Derechos y Deberes Constitucionales, el Consejo de Seguridad Nacional y el procedimiento de Reforma Constitucional.

El proceso de transición tendrá que empezar a caminar por la vía del consenso de la mayor parte de las fuerzas politicas, la exigencia de las cifras lo piden así y los excesivos peligros en el camino deben ser contrarrestados con todos los apoyos posibles en cada uno de los pasos que se vayan dando. Es necesario empezar a abrir todas las mesas de negociación, incluidas las fuerzas armadas y los sectores económicos más reacios al proceso.

La estabilidad del proceso exige abrir este acuerdo a los sectores independientes y a la derecha dialogante; no se debe olvidar que estos sectores representan un 40 por 100 de los votantes. Por esta razón es imprescindible que estos grupos revisen los errores cometidos en la campaña y encuentren un liderazgo lógico y natural. Esta cuestión es imprescindible para la salud del proceso de transición. La representación lógica de la derecha, histórica y numéricamente, debería ser Renovación Nacional y en ella hay lideres que como Allamand han prometido una oposición fiel al Gobierno de Aylwin. 
El eje principal, que no exclusivo, de este consenso y del proceso de transición lo constituyen la Democracia Cristiana y Renovación Nacional, ahora bien la DC como fuerza mayoritaria debe tener en cuenta el peso que la izquierda tiene en el país. Los primeros pasos dados por Aylwin al nombrar en su Gobierno seis ministros socialistas (dos ministerios de extrema importancia como son el de Economia, para Carlos Ominami, y el de Educación, para Ricardo Lagos), es sólo una muestra de lo que tiene que ser una práctica continuada para obtener el apoyo por la izquierda al proceso. Un desplazamiento a la derecha de la DC, aunque de mayor rentabilidad para el Partido Socialista a medio o largo plazo, crearía distorsiones que pondrían en grave riesgo el proceso de transición en estos cuatro años.

La izquierda en Chile ha jugado y debe jugar un papel primordial en el proceso de transición. El Partido Socialista como fuerza mayoritaria dentro de la izquierda, aunque dividida, ha sido la clave estratégica primordial en este proceso. En la ilegalidad fue capaz de crear dos coaliciones Ilamadas "partidos instrumentales»: el PPD (creado por Lagos donde se encuentran los socialistas de Núñez y Arrate) y el PAIS (socialistas de Almeyda). En estos partidos se agruparon otras fuerzas políticas y sectores independientes, siendo el principal lazo de unión la lucha por la democracia frente a la dictadura. EI PAIS unió a socialistas junto con católicos de izquierda e hizo posible la acomodación del Partido Comunista y del MIR al proceso democrático. El PPD agrupó a socialistas, radicales, humanistas, católicos, socialdemócratas y creó un frente amplio que ha obtenido un considerable contingente de votos, siendo su líder Ricardo Lagos el candidato más votado de la izquierda.

Los logros del socialismo en el proceso de transición son muy satisfactorios, pero sería necesario que realizase una acomodación a la nueva realidad que se abre en marzo. Para ello debería iniciar un proceso rápido de maduración que pasa necesariamente por acabar de una vez la unidad frustrada tantas veces. El socialismo ha perdido mucho tiempo frente a la Democracia Cristiana por circunstancias históricas y desencuentros que todos conocemos, pero es necesario en el proceso de transición e imprescindible en el sistema de partidos, un recambio político, institucional y de Gobierno por parte de la izquierda.

Los partidos instrumentales cumplieron su función y la pueden seguir cumpliendo, pero en los próximos meses existirá una normalización del sistema de partidos en el sentido lógico de todos los sistemas democráticos, y el apoyo a las labores de Gobierno presentes y futuras pasa inevitablemente por una organización socialista unida y fuerte, independientemente del futuro de otras fórmulas. Ricardo Lagos, lider del PPD, tercera mayoría nacional, está obligado a asumir esta responsabilidad y a jugar un papel principal en el proceso de unidad. 
Sería una contradicción histórica tener seis ministros en el Gobierno careciendo de un Partido Socialista unido. De igual forma es imprescindible acabar cuanto antes las discusiones programático e ideológicas que propicien la unidad, para pasar inmediatamente a diseñar programas y políticas de Gobierno. No obstante, el proceso de maduración de un partido está sometido a unos pasos y ritmos que no se deben forzar si no se quiere pagar en el futuro precios más altos, pero tampoco es momento para hacer de cuestiones coyunturales requisitos imprescindibies para la unidad.

La declaración doctrinaria dentro del Protocolo de Unidad, aprobado el 29 de diciembre, parece adecuada no sólo para propiciar la unidad de las dos corrientes socialistas, sino también para incorporar o, en su caso, facilitar acuerdos concretos con los otros partidos y grupos que hoy se encuentran en los "partidos instrumentales".

Lo complejo de las reformas constitucionales, las dificultades dentro del sistema de partidos, la sombra de Pinochet y el papel de las fuerzas armadas, la satisfacción de exigencias sociales que en algunas cuestiones son irrenunciables, auguran un proceso de transición complejo y lleno de peligros. La decisión y prudencia del Presidente Aylwin y de los partidos implicados, junto a la madurez política de este puelbo, serán la clave para llevar a buen fin el proceso de transición a un sistema democrático en Chile. 